\title{
Nose-down saddle tilt improves gross efficiency during seated-uphill cycling
}

\author{
Ross D. Wilkinson ${ }^{* 1}$ and Rodger Kram ${ }^{1}$ \\ ${ }^{1}$ Locomotion Laboratory, Department of Integrative Physiology, University of Colorado, Boulder, CO, USA
}

July 16, 2021

\begin{abstract}
Riding uphill presents a challenge to competitive and recreational cyclists. Based on only limited evidence, some scientists have reported that tilting the saddle nose down improves uphillcycling efficiency by as much as 6\%. Purpose: Here, we investigated if simply tilting the saddle nose-down increases efficiency during uphill cycling, which would presumably improve performance. Methods: Nineteen healthy, recreational cyclists performed multiple 5-min trials of seated cycling at $\sim 3 \mathrm{~W} \mathrm{~kg}^{-1}$ on a large, custom-built treadmill inclined to $8^{\circ}$ under two saddletilt angle conditions: parallel to the riding surface and $8^{\circ}$ nose down. We measured subjects' rates of oxygen consumption and carbon dioxide production using an expired-gas analysis system and then calculated their average metabolic power during the last two min of each 5-min trial. Results: We found that, compared to the parallel-saddle condition, tilting the saddle nose down by $8^{\circ}$ improved gross efficiency from 0.205 to 0.208 - an average increase of $1.4 \pm 0.2 \%, \mathrm{t}_{18}=5.9$, $\mathrm{p}<.001, \mathrm{CI}_{95 \%}[0.9,1.9], \mathrm{ES}=1.3$. Conclusion: Our findings are relevant to competitive and recreational cyclists and present an opportunity for innovating new devices and saddle designs that enhance uphill-cycling efficiency. The effect of saddle tilt on other slopes and the mechanism behind the efficiency improvement remain to be investigated.
\end{abstract}

\footnotetext{
${ }^{*}$ Corresponding Author: Ross D. Wilkinson (ross.wilkinson@colorado.edu) All authors have read and approved this version of the manuscript for pre-print. Authors R.D.W. @rd_wilkinson and R.K. @RodgerKram can be reached on Twitter. Please cite as: Wilkinson, R.D., \& Kram, R. (2021). Nose-down saddle tilt improves gross efficiency during seated-uphill

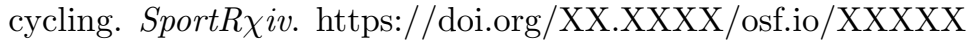




\section{Abbreviations}

ES Effect size

GE Gross efficiency

RER Respiratory exchange ratio

SEM Standard error of the mean

UCI Union Cycliste Internationale

$\dot{\mathrm{VO}}_{2}$ Rate of oxygen uptake 


\section{Introduction}

Uphill cycling often determines the winners of races like the Tour de France, and recreational cyclists also find hills a special challenge. Based on limited evidence, some scientists [1, 2] and cyclists [3, 4] report that tilting the saddle nose down improves both performance and comfort when cycling uphill. Here, we investigated if simply tilting the saddle nose down can increase metabolic efficiency during uphill cycling, which would presumably improve performance.

In cycling, as the uphill slope of the riding surface increases, a larger portion of the gravitational force resists overall forward motion and acts to pull the rider off the back of the saddle. Three forces can prevent the rider from sliding off the saddle: saddle/clothing friction, pedal applied forces, and handlebar applied forces. Friction forces are metabolically free and thus preferable. Regarding pedal forces, as the crank passes top dead center $\left(0^{\circ}\right)$, the reaction to rider-applied tangential pedal forces acts to push the rider backwards off the saddle and as the crank passes bottom dead center $\left(180^{\circ}\right)$ the pedal reaction force acts to pull the rider forward on the saddle. Leg muscle activation patterns during seated cycling on level, $5.7^{\circ}$, and $11.3^{\circ}$ slopes suggest that riders may ingeniously adapt their pattern of pedal force application on steeper uphill slopes [5]. For example, on $5.7^{\circ}$ and $11.3^{\circ}$ slopes, subjects decreased their knee-extensor muscle (i.e., rectus femoris) activity by $13.9 \%$ and $25.3 \%$, respectively. Those changes occurred predominantly as the crank passed over top dead center. Additionally, subjects significantly increased their hamstring (i.e., biceps femoris) activity as the crank passed through bottom dead center. The potential benefit of these adaptations in muscle activity is that tangential pedal force applied as the crank passes bottom dead center generates mechanical power and the reaction force pulls the rider forward on the saddle. While suggestive, those data do not establish causality and the concurrent pedal forces were not reported. Actively pulling backwards on the handlebars can also counteract sliding backwards on the saddle but presumably incurs a metabolic cost.

Another way to passively prevent sliding backwards off the saddle during uphill cycling may be to tilt the saddle nose down. Fonda et al. [6] used an adjustable seat post, which both steepened the effective seat tube angle and tilted the saddle nose down. Their results showed that the device mitigated the previously described changes in leg-muscle activity due to slope, increased perceived performance, and reduced perceived exertion. Fonda and Sarabon [2] also mention having pilot data using the same adjustable seat post that indicated a remarkable $6 \%$ reduction in oxygen consumption and a 30-60\% reduction in elbow-flexor muscle activity (i.e., m. brachioradialis). Unfortunately, they did not provide any other details. Those results are tantalizing, but more evidence is needed to establish if there is an effect of nose-down saddle tilt on uphill-cycling efficiency. Here, we tested the null hypothesis that when cycling up an $8^{\circ}$ slope, tilting the saddle $8^{\circ}$ nose down would have no effect on gross efficiency.

\section{Methods}

Nineteen healthy recreational cyclists volunteered (18 males/1 female, age: $30.8 \pm 9.3$ years, height: $1.80 \pm 0.06 \mathrm{~m}$, mass: $74.0 \pm 11.3 \mathrm{~kg}$, mean \pm one standard deviation). Subjects were between 18-49 years old, in good health, cycled $>4$ hours per week, and used their own clip-in pedals and shoes. All subjects gave written informed consent as per the University of Colorado Boulder Institutional Review Board and in accordance with the Declaration of Helsinki.

All subjects rode the same 56-cm road bicycle (Roubaix, Specialized Bicycle Components Inc., Morgan Hill, CA, USA). We adjusted saddle height to each subject's personal preference and maintained tire pressure at 100 psi $(689 \mathrm{kPa})$. The bike was equipped with a crank-based mechanical power meter (Quarq DZero, SRAM, Corp. Chicago, IL, USA) with $172.5 \mathrm{~mm}$ long cranks. The power 
meter samples crank angular velocity and torque at $65 \mathrm{~Hz}$ and then transmits 1-second averages of cadence and power to the Garmin head unit (Edge 1000, Garmin Ltd, Olathe, KA, USA). As per the manufacturer's recommendations, we zeroed the power meter prior to each testing session.

Subjects rode on a large custom-built, motorized treadmill (length $3.2 \mathrm{~m}$, width $0.9 \mathrm{~m}$ ) inclined to a slope of $8.0^{\circ}(14.1 \%)$. We chose that angle based on pilot testing indicating $8.0^{\circ}$ was the steepest slope possible while maintaining a cadence that was still comfortable for riding in a seated posture. Prior to the experimental trials, subjects practiced cycling uphill on the treadmill until they gave verbal confirmation that they felt safe and confident. Riders wore a helmet and a safety harness that did not apply any impeding, aiding, or lifting force to the rider.

We weighed each subject and calculated the treadmill belt velocity $\left(V_{b}\right)$ needed to attain the target mechanical power output of $3 \mathrm{~W} \mathrm{~kg}^{-1}$ of body mass $\left(P_{m} e c h\right)$ using Equation 1.

$$
V_{b}=\frac{P_{m e c h} \cdot E_{d t}}{\left(m_{r}+m_{b}\right) \cdot g(\sin (\theta)+\cos (\theta) \cdot \mu)}
$$

$E_{d t}$ is the presumed efficiency of the drivetrain (0.976) [7], $m_{r}$ is the measured rider mass, $m_{b}$ is the measured bicycle mass, $\mathrm{g}$ is $9.81 \mathrm{~m} \mathrm{~s}^{-2}, \theta$ is the measured slope of the treadmill, and $\mu$ is the measured coefficient of rolling resistance (0.0032). We measured the treadmill belt velocity using a contact tachometer (DT-107A, Shimpo, Cedarhurst, NY, USA). Due to the sensitivity of Equation 1 to changes in rider mass, we re-weighed the subject in between trials, and they drank enough water to maintain their pre-experiment body mass for each trial. Subjects next completed a 5-min familiarization trial of uphill cycling at $\sim 3 \mathrm{~W} \mathrm{~kg}^{-1}$ while breathing through the open-circuit, expiredgas analysis system (TrueOne 2400, ParvoMedics, Sandy, UT, USA). Then, they rested for $5 \mathrm{~min}$ before beginning the experimental trials.

Subjects performed four, 5-min trials of seated cycling at $\sim 3 \mathrm{~W} \mathrm{~kg}^{-1}$ with 5-min rest between each. The four trials consisted of two replicates of two saddle tilt angle conditions: parallel (P) to the riding surface and $8^{\circ}$ nose down (ND), which the subjects completed in a mirrored order. That is, either P, ND, ND, P or ND, P, P, ND. The order assignment was counterbalanced across subjects and randomized. Due to the steep slope, all subjects chose to ride in the "easiest" gear combination (lowest ratio) available (30x42).

During the experimental trials, we measured each subject's rate of oxygen consumption and carbon dioxide production using the expired-gas analysis system. We then calculated their metabolic power $[8,9]$ from the rates of oxygen consumption and carbon dioxide production during the last two min of each 5-min trial. We calculated gross efficiency (GE) as the ratio of mechanical power output to gross metabolic power consumption [10]. We calculated the percentage change in GE during the nose-down condition compared to the parallel condition as the mean difference divided by GE in the parallel condition.

\subsection{Statistical analysis}

We used a paired t-test to compare key variables between the two conditions but only after a JarqueBera test with Monte Carlo simulation confirmed that the distribution of the pairwise differences matched a normal distribution. For each outcome measure, we analyzed each subject's mean from the two replicates of the two conditions. An a-priori power analysis [11], determined that a sample size of 19 would be required to detect our smallest effect size of interest (0.48) and that we could detect effect sizes $\geq 0.8$ with $90 \%$ power at $\alpha=0.05$. We corrected p-values using the Dunn-Sidak method. For statistical comparisons, we provide the effect size (ES) as Hedges' $\mathrm{g}_{a v}$. We present the results from the two experimental conditions as the group mean \pm the standard error of the mean (SEM). 


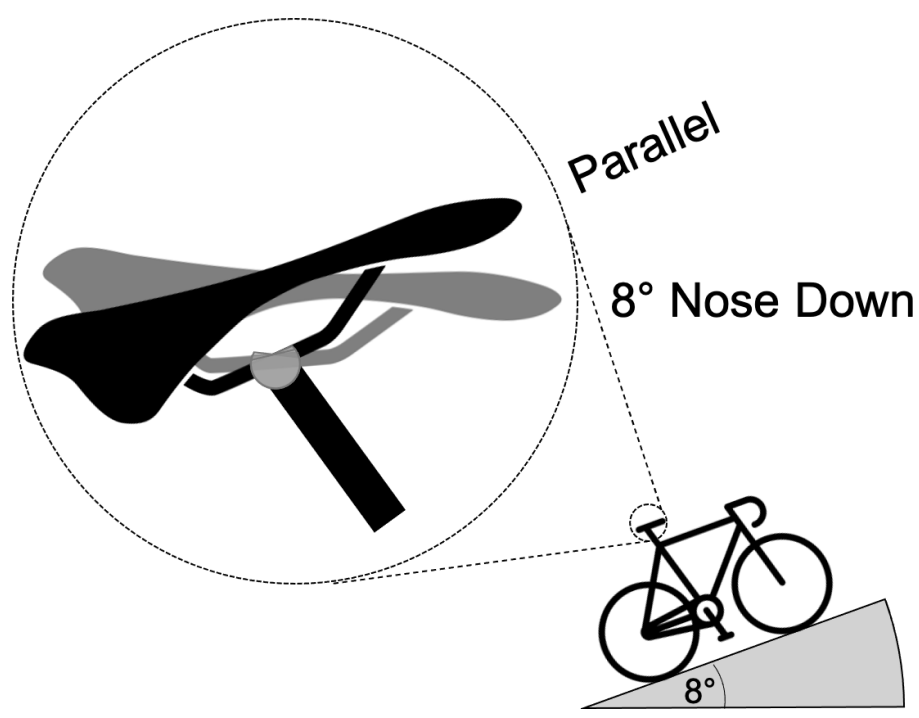

Figure 1: Diagram illustrating saddle-tilt angle in the two seated-cycling conditions on a treadmill inclined to a slope of $8^{\circ}(14.1 \%)$.

\section{Results}

Compared to the parallel-saddle condition, tilting the saddle nose down by $8^{\circ}$ improved gross efficiency from 0.205 to 0.208 - an average increase of $1.4 \pm 0.2 \%, \mathrm{t}_{18}=5.9, \mathrm{p}<.001, \mathrm{CI}_{95 \%}[0.9,1.9], \mathrm{ES}=1.3$ (see Table 1). Thus, we reject our null hypothesis. In our sample, 16 of 19 subjects exhibited increased efficiency. Based on the probability distribution of our data (see Figure 2B), $\sim 91 \%$ of cyclists are likely to improve their gross efficiency by tilting the saddle nose down by $8^{\circ}$ when cycling on an $8^{\circ}$ slope. Furthermore, $\sim 27 \%$ of cyclists are likely to experience a $>2 \%$ improvement in gross efficiency. The mean mechanical power output, cadence, and RER were virtually identical between the two conditions (see Table 1).
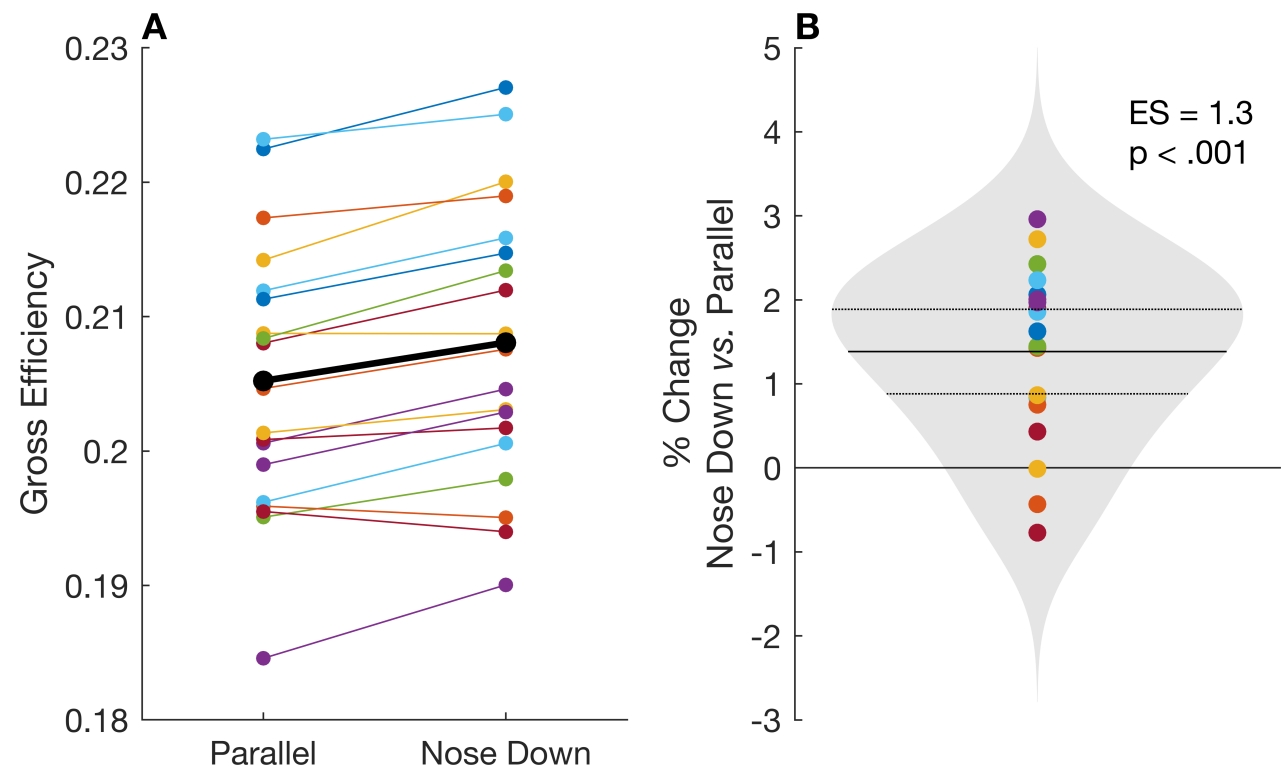

Figure 2: A. Individual (color) and group mean (black) gross efficiency. B. A violin plot [12] of the individual (color) and group mean (solid line) percentage change in gross efficiency. The grey patch shows the probability distribution of the percentage changes, and the dotted lines show the $95 \%$ confidence intervals. ES, effect size. 


\begin{tabular}{lll}
\hline & Parallel & Nose Down \\
\hline Mechanical Power $\left(\mathrm{W} \mathrm{kg}^{-1}\right)$ & $2.87 \pm 0.05$ & $2.88 \pm 0.05$ \\
Cadence $(\mathrm{RPM})$ & $59.2 \pm 1.0$ & $59.2 \pm 1.0$ \\
$\mathrm{RER}$ & $0.90 \pm 0.01$ & $0.90 \pm 0.01$ \\
$\mathrm{VO}_{2}\left(\mathrm{ml} \mathrm{kg}^{-1} \mathrm{~min}^{-1}\right)$ & $40.4 \pm 0.6$ & $39.9 \pm 0.6$ \\
Metabolic Power $\left(\mathrm{W} \mathrm{kg}^{-1}\right)$ & $14.0 \pm 0.2$ & $13.8 \pm 0.2$ \\
Gross Efficiency & $0.205 \pm 0.002$ & $0.208 \pm 0.002$ \\
\hline
\end{tabular}

Table 1: Group mean $( \pm$ SEM) mechanical and metabolic data during seated cycling on a treadmill inclined to a slope of $8^{\circ}$ either with the saddle angle parallel to the treadmill surface or tilted $8^{\circ}$ nose down.

\section{Discussion}

Tilting the saddle nose down by $8^{\circ}$ when cycling up an $8^{\circ}$ slope improved gross efficiency by $1.4 \%$ and thus, we reject our null hypothesis. To the best of our knowledge, this is the first study to provide clear evidence that merely tilting the saddle nose down can improve a rider's gross efficiency during seated uphill cycling.

At this point, we cannot provide any of our own evidence regarding the mechanism that underlies the improvement in gross efficiency. The pilot data of [2] suggests the mechanism may be a reduction in upper-body muscle activity, specifically elbow-flexor activity. Presumably most of the metabolic energy is consumed by the legs, but a large reduction in elbow-flexor activity might be enough to account for a $1.4 \%$ overall reduction in metabolic power. Further research is required to determine if there is a causal association between changes in electromyographic activity of upper-body muscles and the improvements in gross efficiency.

Our results cannot yet be generalized to all slopes and all power outputs, since we only matched the nose-down tilt angle to a single slope of $8^{\circ}$ and at a single power output of $\sim 3 \mathrm{~W} \mathrm{~kg}^{-1}$. We postulate that tilting the saddle nose down would have a smaller effect on shallower slopes and a larger effect on steeper slopes due to the increase in gravitational force acting to pull the rider off the saddle. It is difficult to speculate on whether the effect of nose-down saddle tilt would be dependent on power output. If the mechanism is a reduction in elbow-flexor activity, due to reduced handlebar forces, then the effect would likely depend on pedal force rather than power output. Thus, a rider may experience greater improvements in gross efficiency when greater crank torques are required. Further research is needed to confirm whether efficiency improvements are dependent on slope and/or power output.

Another possible benefit of tilting the saddle nose down may be a reduction in cycling-induced discomfort and pathologies related to postural adjustments and saddle pressure. Salai et al. [1] provided evidence that tilting the saddle nose down by $10-15^{\circ}$ reduces lower-back pain and speculated that the tilt decreased forces on lumbar vertebrae by helping riders maintain a more neutral spine. Sequenzia et al. [13] investigated if a variable-geometry saddle prototype, which allowed riders to adjust their nose-down saddle-tilt angle, had neurological benefits. They found that when cycling up an $\sim 8.5^{\circ}$ slope, nose-down saddle tilt maintained non-pathological latency and sensory conduction velocity in the pudendal nerve. Longitudinal clinical studies would be required to ascertain if tilting the saddle nose down during uphill cycling can reduce cycling-induced pathologies.

How much might a 1.4\% improvement in efficiency affect cycling performance? It is important to first note that due to aerodynamic resistance, a $1.4 \%$ increase in efficiency does not translate to a $1.4 \%$ increase in cycling velocity. During level-ground cycling, the mechanical power required to 
overcome aerodynamic drag increases with the third power of velocity [7]. Thus, at $50 \mathrm{~km} \mathrm{~h}^{-1}(\sim 13.9$ $\mathrm{m} \mathrm{s}^{-1}$ ), a $1.4 \%$ increase in efficiency might increase velocity by only $0.5 \%$. During steep uphill cycling, overcoming gravity dominates the total mechanical power required but aerodynamic resistance is not insignificant for elite cyclists. For example, the most cited record time for ascending Alpe d'Huez, a famous 13.8- $\mathrm{km}$ climb with an average slope of $8.1 \%\left(4.6^{\circ}\right)$, is $37 \mathrm{~min} 35 \mathrm{~s}$ - an average velocity of 22 $\mathrm{km} \mathrm{h}^{-1}\left(6.12 \mathrm{~m} \mathrm{~s}^{-1}\right)$. For a $57-\mathrm{kg}$ rider, with a 7 -kg bicycle, on a slope of $8.1 \%$, overcoming gravity and rolling resistance would require $332 \mathrm{~W}$ of mechanical power while overcoming aerodynamic resistance would require an additional $32 \mathrm{~W}[7]$. With metabolic power consumption held constant, if a rider could produce $1.4 \%$ more mechanical power, due to an improvement in efficiency, their velocity would increase to $22.3 \mathrm{~km} \mathrm{~h}^{-1}\left(6.19 \mathrm{~m} \mathrm{~s}^{-1}\right)-$ a $1.2 \%$ increase. We calculate that an elite cyclist riding in a seated posture, could save $\sim 26 \mathrm{~s}$ on the Alpe d'Huez climb by tilting their saddle nose down. For slower, recreational cyclists, the increase in ground velocity would be nearer to $1.4 \%$ and they would save significantly more time. See Appendix 1 for details of these calculations.

Our results have particular significance for cyclists who compete in Union Cycliste Internationale (UCI) sanctioned road-racing events. In 2016, the UCI changed their rules regarding the maximum saddle-tilt angle allowed during road-cycling events [14]. The new rule allows a maximum saddle angle of $9^{\circ}$ from horizontal. Our results suggest that riders could significantly improve their gross efficiency when cycling on steep slopes $\left(>8^{\circ}\right)$ by tilting their saddle nose down to match the slope. Such steep slopes are rare in road cycling but common in mountain biking and the UCI rules for mountain biking do not restrict saddle tilt.

Our findings suggest an opportunity to innovate new devices and saddle designs that enhance uphillcycling efficiency. However, it may be prudent to wait until evidence emerges about the generalizability of our findings. Elucidating the underlying mechanism(s) may point to robust designs that can improve uphill-cycling efficiency without potentially hampering efficiency in other scenarios. Specifically, the UCI rules define saddle tilt based on the highest points at the front and rear of the saddle. A U-shaped saddle which is lowest in the middle but has high points at the front and rear-like an equestrian saddle - would be UCI legal and still may provide substantial benefits on steep slopes.

In conclusion, we find that tilting the saddle nose down by $8^{\circ}$ on an $8^{\circ}$ slope improves gross efficiency by an average of $1.4 \%$ compared to having the saddle parallel to the slope. We intend to and encourage others, to expand on these findings by testing the effect on other slopes and investigating the underlying mechanism(s).

\section{Contributions}

Conception and design: R.D.W., R.K.; Acquisition of data: R.D.W., R.K.; Analysis and interpretation of data: R.D.W., R.K.; Drafted and/or revised the manuscript: R.D.W., R.K.; Approved final version of the manuscript: R.D.W., R.K.

\section{Funding Information}

Supported by an unrestricted gift from Koch-Bein, LLC.

\section{Conflict of interest}

Koch-Bein, LLC have a patent pending for a saddle-related mechanism. Our laboratory also receives unrestricted financial gifts from Specialized Bicycle Components, Inc., but they did not commission 
or fund this specific study.

\section{Data availability}

Data can be accessed at https://osf.io/56jxu/.

\section{Appendix 1}

\begin{tabular}{|c|c|c|c|c|}
\hline & Elite 1 & Elite 2 & Rec. 1 & Rec. 2 \\
\hline \multicolumn{5}{|l|}{ Power and efficiency } \\
\hline Metabolic power (W) & 1580 & $=$ & 1125 & $=$ \\
\hline Gross efficiency & 0.2300 & 0.2332 & 0.2000 & 0.2028 \\
\hline \multicolumn{5}{|l|}{ Rider and equipment } \\
\hline Body mass (kg) & 57 & $=$ & 75 & $=$ \\
\hline Bike mass (kg) & 7 & $=$ & 10 & $=$ \\
\hline Frontal surface area drag factor & 0.2565 & $=$ & $=$ & $=$ \\
\hline Coefficient of rolling resistance & 0.0032 & $=$ & $=$ & $=$ \\
\hline Spoke drag factor & 0.0044 & $=$ & $=$ & $=$ \\
\hline \multicolumn{5}{|l|}{ Environment } \\
\hline Gravity $\left(\mathrm{m} \mathrm{s}^{-2}\right)$ & 9.81 & $=$ & $=$ & $=$ \\
\hline Slope $(\%)$ & 8.1 & $=$ & $=$ & $=$ \\
\hline Slope $\left(^{\circ}\right)$ & 4.6 & $=$ & $=$ & $=$ \\
\hline Distance $(\mathrm{km})$ & 13.8 & $=$ & $=$ & $=$ \\
\hline Air density $\left(\mathrm{kg} \mathrm{m}^{-3}\right)$ & 1.06 & $=$ & $=$ & $=$ \\
\hline Wind velocity $\left(\mathrm{m} \mathrm{s}^{-1}\right)$ & 0 & $=$ & $=$ & $=$ \\
\hline \multicolumn{5}{|l|}{ Results } \\
\hline Mechanical power (W) & 363.4 & 368.5 & 225.0 & 228.2 \\
\hline Mechanical power $\left(\mathrm{W} \mathrm{kg}^{-1}\right)$ & 6.38 & 6.46 & 3.00 & 3.04 \\
\hline Gain in mech. power $(\%)$ & - & 1.40 & - & 1.40 \\
\hline Overground velocity $\left(\mathrm{m} \mathrm{s}^{-1}\right)$ & 6.115 & 6.187 & 3.075 & 3.117 \\
\hline Gain in velocity (\%) & - & 1.17 & - & 1.33 \\
\hline Finishing time (h:mm:ss) & $0: 37: 37$ & $0: 37: 11$ & $1: 14: 48$ & $1: 13: 47$ \\
\hline Time saving (s) & - & 26 & - & 61 \\
\hline
\end{tabular}

Table 2: Translation of a $1.4 \%$ improvement in metabolic efficiency to time savings on Alpe d'Huez for an exemplar elite and recreational (Rec.) rider. We used the equations from Martin et al. [7] to estimate overground velocity. 


\section{References}

[1] Moshe Salai, Tamar Brosh, Alexander Blankstein, Arial Oran, and Aharon Chechik. Effect of changing the saddle angle on the incidence of low back pain in recreational bicyclists. British Journal of Sports Medicine, 33(6):398-400, 1999. doi: 10.1136/bjsm.33.6.398.

[2] Borut Fonda and Nejc Sarabon. Biomechanics and energetics of uphill cycling: A review. Kinesiology, 44(1):5-17, 2012.

[3] PedalChile. Best saddle angle for climbing, 2020. URL https://pedalchile.com/blog/ climbing-saddle-tilt.

[4] PinkBike. Opinion: Why is everyone talking about seat tube angles?, 2019. URL https://www . pinkbike.com/news/opinion-why-is-everyone-talking-about-seat-tube-angles.html.

[5] Nejc Sarabon, Borut Fonda, and Goran Markovic. Change of muscle activation patterns in uphill cycling of varying slope. European Journal of Applied Physiology, 112(7):2615-2623, 2012. doi: 10.1007/s00421-011-2236-1.

[6] Borut Fonda, Andrej Panjan, Goran Markovic, and Nejc Sarabon. Adjusted saddle position counteracts the modified muscle activation patterns during uphill cycling. Journal of Electromyography and Kinesiology, 21(5):854-860, 2011. doi: 10.1016/j.jelekin.2011.05.010.

[7] James C. Martin, Douglas L. Milliken, John E. Cobb, Kevin L. McFadden, and Andrew R. Coggan. Validation of a mathematical model for road cycling power. Journal of Applied Biomechanics, 14 (3):276-291, 1998.

[8] Shalaya Kipp, William C. Byrnes, and Rodger Kram. Calculating metabolic energy expenditure across a wide range of exercise intensities: The equation matters. Applied Physiology, Nutrition and Metabolism, 43(6):639-642, 2018. doi: 10.1139/apnm-2017-0781.

[9] F Peronnet and D Massicotte. Table of non-protein respiratory quotient: An update. Canadian Journal of Sport Science, 16(4):23-29, 1991.

[10] Glenn A. Gaesser and George A. Brooks. Muscular efficiency during steady-rate exercise: Effects of speed and work rate. Journal of Applied Physiology, 38(6):1132-1139, 1975.

[11] Franz Faul, Edgar Erdfelder, Albert-Georg Lang, and Axel Buchner. G*power 3: A flexible statistical power analysis program for the social, behavioral, and biomedical sciences. Behaviour Research Methods, 39(2):175-191, 2007.

[12] Jerry L Hintze and Ray D Nelson. Violin plots: A box plot-density trace synergism statistical computing and graphics violin plots: A box plot-density trace synergism. The American Statistician, 52(2):181-184, 1998.

[13] G. Sequenzia, G. Fatuzzo, S. M. Oliveri, and R. Barbagallo. Interactive re-design of a novel variable geometry bicycle saddle to prevent neurological pathologies. International Journal on Interactive Design and Manufacturing, 10(2):165-172, 2016. doi: 10.1007/s12008-015-0293-0.

[14] Union Cycliste Internationale. Clarification Guide of the UCI Technical Regulation (Version 17.01.2021). Technical report. URL https://www.uci.org/docs/default-source/equipment/ clarificationguideoftheucitechnicalregulation-2018-05-02-eng_english.pdf?sfvrsn= fd56e265_92. 